\title{
Superconducting Gap Anisotropy and Quasiparticle Interactions: A Doping Dependent Photoemission Study
}

\author{
J. Mesot,,${ }^{1,2}$ M. R. Norman, ${ }^{1}$ H. Ding, ${ }^{3}$ M. Randeria, ${ }^{4}$ J. C. Campuzano, ${ }^{1,2}$ A. Paramekanti, ${ }^{4}$ H. M. Fretwell, ${ }^{2}$ \\ A. Kaminski, ${ }^{2}$ T. Takeuchi, ${ }^{5}$ T. Yokoya, ${ }^{6}$ T. Sato, ${ }^{6}$ T. Takahashi, ${ }^{6}$ T. Mochiku, ${ }^{7}$ and K. Kadowaki ${ }^{8}$ \\ ${ }^{1}$ Materials Sciences Division, Argonne National Laboratory, Argonne, Illinois 60439 \\ ${ }^{2}$ Department of Physics, University of Illinois at Chicago, Chicago, Illinois 60607 \\ ${ }^{3}$ Department of Physics, Boston College, Chestnut Hill, Massachusetts 02467 \\ ${ }^{4}$ Tata Institute of Fundamental Research, Mumbai 400005, India \\ ${ }^{5}$ Department of Crystalline Materials Science, Nagoya University, Nagoya 464-01, Japan \\ ${ }^{6}$ Department of Physics, Tohoku University, 980-8578 Sendai, Japan \\ ${ }^{7}$ National Research Institute for Metals, Sengen, Tsukuba, Ibaraki 305, Japan \\ ${ }^{8}$ Institute of Materials Science, University of Tsukuba, Ibaraki 305, Japan
}

(Received 23 December 1998)

\begin{abstract}
Comparing photoemission measurements on Bi2212 with penetration depth data, we show that a description of the nodal excitations of the $d$-wave superconducting state in terms of noninteracting quasiparticles is inadequate, and we estimate the magnitude and doping dependence of the Landau interaction parameter which renormalizes the linear $T$ contribution to the superfluid density. Furthermore, although consistent with $d$-wave symmetry, the gap with underdoping cannot be fit by the simple $\cos k_{x}-\cos k_{y}$ form, which suggests an increasing importance of long range interactions as the insulator is approached.
\end{abstract}

PACS numbers: 74.25.Jb, 71.18.+y, 74.72.Hs, 79.60.Bm

There is little doubt about the fundamental importance of many-body interactions in high temperature cuprate superconductors [1]. Quantifying these interactions is difficult in the normal state of these materials, given the lack of well-defined single-particle excitations as revealed by various experiments. On the other hand, well-defined quasiparticle excitations do exist in the superconducting state, and it is believed that a description of the low temperature state in terms of superfluid Fermi liquid theory is appropriate. In Fermi liquid theory, the quasiparticles are characterized by a renormalized Fermi velocity $v_{F}$, and their residual interactions described by Landau parameters, which manifest themselves through a renormalization of various response functions relative to that given by a noninteracting theory. For example, in the cuprates, the Fermi velocity $v_{F}$ has been determined by angle resolved photoemission studies (ARPES) in $\mathrm{Bi}_{2} \mathrm{Sr}_{2} \mathrm{CaCu}_{2} \mathrm{O}_{8+\delta}$ (Bi2212) [2] to be renormalized by a factor of 2 to 3 over that given by band theory. The strong renormalization of the superfluid density $\rho_{s}(0)$ has also been known for some time, where one sees a scaling with the number of doped holes: the Uemura relation [3].

In this paper we examine an issue which is at the heart of the nature of quasiparticles in the superconducting state of the cuprates; that is, whether the slope of the superfluid density at low temperatures, $d \rho_{s} / d T$, is affected by interactions or not, and what the relation of its renormalization is to that of $\rho_{s}(0)$, questions of considerable debate in the recent literature [4,5]. The importance of $\rho_{s}(T)$ to an understanding of cuprate superconductivity derives from the early observation of a linear $T$ suppression of $\rho_{s}(T)$ [6], since this is explained most naturally by the thermal exci- tations of quasiparticles near the nodes of a $d$-wave superconducting gap. Related to this is the interesting question of whether the gap around the node scales with $T_{c}$, as has been suggested from a recent analysis of magnetic penetration depth data [7].

To address these issues we use the unique capability of ARPES to directly measure the Fermi wave vector $k_{F}$, velocity $v_{F}$, and the superconducting gap anisotropy near the node, from which we can estimate the slope of $\rho_{s}(T)$ assuming noninteracting quasiparticles. Comparing this with the actual value obtained by penetration depth experiments leads to a direct estimate of the renormalization due to quasiparticle interactions. This is done by exploiting the relation [5],

$$
\left|\frac{d \rho_{s}}{d T}(T=0)\right| \propto\left|\frac{d}{d T}\left(\frac{1}{\lambda^{2}}\right)\right|=A \beta^{2} \frac{v_{F} k_{F}}{v_{\Delta}},
$$

where $\lambda$ is the penetration depth, and $A$ is a doping independent constant: $A=4 \ln 2 \alpha k_{B} n / c d$ with $\alpha$ the fine structure constant, $k_{B}$ the Boltzmann constant, $c$ the speed of light, and $n$ the number of $\mathrm{CuO}_{2}$ layers (four for Bi2212) per $c$-axis lattice constant $d$ (30.9 $\AA$ for Bi2212). ARPES is used to determine the three parameters at the node: the Fermi velocity $\boldsymbol{v}_{F}$, the Fermi wave vector $k_{F}$, and the slope of the superconducting gap $v_{\Delta}=$ $1 / 2|d \Delta / d \phi|(\phi=\pi / 4)$, where $\phi$ is the Fermi surface angle. The latter is normalized such that $v_{\Delta}=\Delta_{\max }$ for the simple $d$-wave gap $\Delta(\phi)=\Delta_{\max } \cos (2 \phi)$.

The only unknown in Eq. (1) is the renormalization factor $\beta$ due to quasiparticle interactions; in the isotropic Fermi liquid theory $\beta=1+F_{1 s} / 2$, where $F_{1 s}$ is the 
$l=1$ spin symmetric Landau parameter, and quantifies the backflow of the medium around the quasiparticles [8]. By comparing ARPES and penetration depth data, we estimate $\beta$ and its doping dependence. In particular, different assumptions in the recent literature [4,5,7] about the doping dependence of $v_{\Delta}$ has led to different conclusions regarding the value and doping dependence of $\beta$ in Eq. (1).

Our main results are as follows. (1) We determine the doping dependence of the gap anisotropy from ARPES. Although consistent with a node on the Fermi surface along the zone diagonal $(\phi=\pi / 4)$ for all doping levels, the shape of the gap changes with underdoping: while its maximum value increases [9-11], we find the new result that the gap becomes flatter near the nodes, i.e., $v_{\Delta}$ decreases. (2) Using our data on the doping dependence of $v_{\Delta}$, we exploit Eq. (1) and use available values of the penetration depth $\lambda(T)[6,12-15]$ to estimate the renormalization factor $\beta$. We find that $\beta$ is considerably smaller than unity and decreases with underdoping, in contrast to previous suggestions in the literature $[4,5,7]$. (3) Our results on the doping dependence of the gap anisotropy and its relation to penetration depth data provide important evidence that the strength of both the pairing interaction and the quasiparticle interactions increase with reduced doping.

The ARPES experiments were performed at the Synchrotron Radiation Center, Wisconsin, using both a high-resolution 4-m normal incidence and plane grating monochromators, with a resolving power of $10^{4}$ at $10^{11}$ photons/sec. We used $22 \mathrm{eV}$ photons, with a $17 \mathrm{meV}$ (FWHM) energy resolution, and a momentum window of radius $0.045 \pi$ (in units of $1 / a$ where $a$ is the $\mathrm{Cu}-\mathrm{Cu}$ separation). The high quality single crystal samples were float-zone grown, with doping changed by varying the oxygen partial pressure during annealing. All samples show sharp x-ray diffraction rocking curves and flat surfaces after cleaving as determined from specular laser reflection. We label the samples by their doping (UD for underdoped, OD for overdoped) and onset $T_{c}$.

Figure 1 shows ARPES data at $T=15 \mathrm{~K}$ for an UD75K sample at different $\mathbf{k}$ points along the Fermi surface. $k_{F}$ was carefully chosen using the criterion that the leading edge of the spectrum has minimum binding energy with the steepest slope, when compared with other spectra along a cut perpendicular to the Fermi surface, as discussed earlier [16]. The zero of binding energy $\left(E_{F}\right)$ was determined from the spectra (not shown) of a polycrystalline Pt reference in electrical contact with the Bi2212, recorded at regular intervals to ensure accurate determination of the Fermi energy, $E_{F}$. From the shift of spectral weight away from $E_{F}$, one clearly sees an anisotropic gap, which is maximal near the $(\pi, 0)$ point $(\phi=0)$ and zero near the $(\pi, \pi)$ direction $\left(\phi=45^{\circ}\right)[17,18]$.

For comparison we also plot (dashed line) in Fig. 1 ARPES spectra from an OD87K sample at two points on the Fermi surface. (For more OD data see Ref. [18].) We

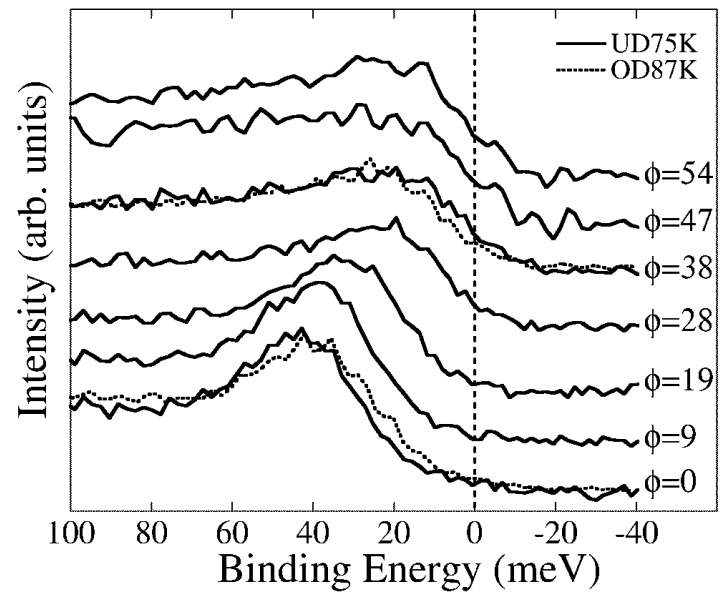

FIG. 1. Spectra of an UD75K Bi2212 sample (solid line) in the vicinity of $E_{F}$ taken at $T=15 \mathrm{~K}$, each labeled by the Fermi surface angle $\phi$. For two angles we also plot spectra from an OD87K sample (dotted line).

immediately see that the UD sample has a larger maximum gap $(\phi=0)$ than the OD one, but it has a smaller gap at the corresponding point $\left(\phi=38^{\circ}\right)$ near the node. Thus the raw data directly give evidence for an interesting change in gap anisotropy with doping.

To quantitatively estimate the gap, we have modeled the low temperature data by a simple BCS spectral function, taking into account the measured dispersion and the known energy and momentum resolutions. Details of this analysis, and error estimates, have been described earlier in the context of OD samples [18,19]. The resulting angular dependence of the gap is plotted in Fig. 2 for six samples.

To further quantify this change in anisotropy, we have used the following expression to fit the gap: $\Delta_{\mathbf{k}}=$ $\Delta_{\text {max }}[B \cos (2 \phi)+(1-B) \cos (6 \phi)]$ with $0 \leq B \leq 1$, where $B$ is determined for each data set. Note that $\cos (6 \phi)$ is the next harmonic consistent with $d$-wave symmetry. We find that while the overdoped data sets are consistent with $B \approx 1$, the parameter $B$ decreases significantly in the underdoped regime. To emphasize the significance of $B<1$, we plot in the panel of an UD75K sample of Fig. 2 a dashed curve with $B=1$ along with the best fit curve for that sample. From these fits, one easily determines the value $v_{\Delta}$ discussed earlier in the context of Eq. (1). In Fig. 3a, we plot $v_{\Delta} / \Delta_{\max }$ for seven samples (the six analyzed above plus an UD85K sample from Ref. [20]). One can clearly see from this figure the trend that underdoping leads to an increase in the maximum gap together with a decrease in the gap slope at the node.

Several questions need to be addressed before proceeding further. First, could the flattening at the node be, in fact, evidence for a "Fermi arc" (a line of gapless excitations), especially since such arcs are seen above $T_{c}$ in the underdoped materials [20]? Given the error bars on gap estimates in Fig. 2, it is impossible to rule out arcs in all the samples. Nevertheless, it is clear that there are samples 


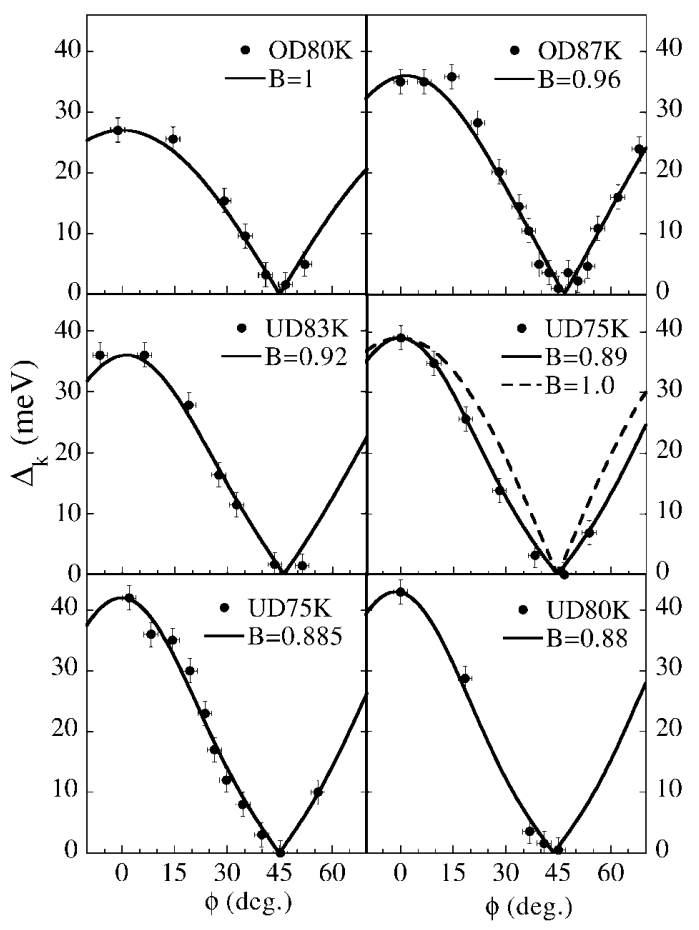

FIG. 2. Values of the superconducting gap as a function of the Fermi surface angle $\phi$ obtained for a series of Bi2212 samples with varying doping. Note two different UD75K samples were measured, and the UD83K sample has a larger doping due to aging [16]. The solid lines represent the best fit using the gap function: $\Delta_{k}=\Delta_{\max }[B \cos (2 \phi)+(1-B) \cos (6 \phi)]$ as explained in the text. The dashed line in the panel of an UD75K sample represents the gap function with $B=1$.

(especially OD87K, UD80K, and UD75K) where there is clear evidence in favor of a point node rather than an arc at low temperatures. Furthermore, it is very important to note that a linear $T$ dependence of $\rho_{s}(T)$ at low temperature, for all doping levels, in clean samples gives independent evidence for point nodes $[6,12,15]$.

Second, is the change in gap anisotropy intrinsic, or related to impurity scattering? We can eliminate the latter explanation on two grounds. The maximum gap increases as the doping is reduced, opposite to what would be expected from pair breaking due to impurities. Also, impurity scattering is expected to lead to a characteristic "tail" to the leading edge [21], for which there is no evidence in the observed spectra (see Fig. 1).

We suggest that the change in the gap function with underdoping is related to an increase in the range of the pairing interaction: the $\cos (6 \phi)$ term in the Fermi surface harmonics can be shown to be closely related to the tight binding function $\cos \left(2 k_{x}\right)-\cos \left(2 k_{y}\right)$, which represents next nearest neighbors interaction, just as $\cos (2 \phi)$ is closely related to the near neighbor interaction $\cos \left(k_{x}\right)-$ $\cos \left(k_{y}\right)$. On very general grounds, the increasing importance of the $\cos (6 \phi)$ term with underdoping could arise from a decrease in screening as one approaches the insulator. Similar effects also arise in specific models. In
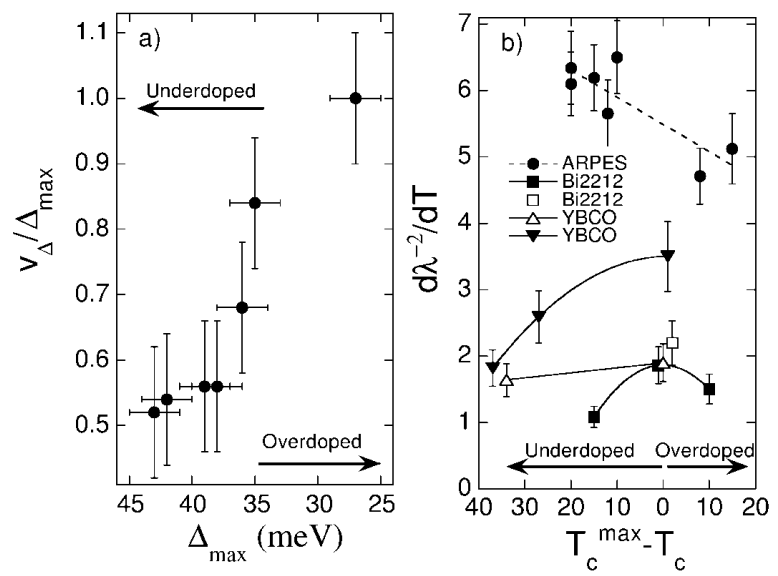

FIG. 3. (a) Normalized slope of the gap at the node $\left(v_{\Delta} / \Delta_{\max }\right)$ vs gap maximum $\Delta_{\max }$. Note the clear drop from unity as one enters the underdoped regime. (b) Slope of the superfluid density (in units of $10^{-9} \AA^{-2} \mathrm{~K}^{-1}$ ) vs $T_{c}^{\max }-T_{c}$ estimated from ARPES measurements based on noninteracting quasiparticles in Bi2212 (filled circles) compared with direct penetration depth measurements in YBCO (open [12] and filled [15] triangles, $T_{c}^{\max }=92 \mathrm{~K}$ ) and Bi2212 (open [13] and filled [14] squares, $\left.T_{c}^{\max }=95 \mathrm{~K}\right)$. The error bars for the latter values were $\pm 15 \%$ based on $\pm 5 \%$ error bars for $\lambda(0)$ [14]. The lines are guides to the eye.

models of spin-fluctuation mediated $d$-wave pairing, an increase in the antiferromagnetic correlation length with underdoping leads to a more sharply peaked pairing interaction in $\mathbf{k}$ space, causing a flattening of the gap around the node as we find here. In interlayer tunneling models, one also expects changes in the shape of the gap which might be correlated with doping [22].

We note that the ratio of the dispersion normal to the Fermi surface $\left(v_{F}\right)$ to that along the Fermi surface $\left(v_{\Delta}\right)$ is quite large, 20 in the overdoped case, and becomes even larger as the doping decreases, in contrast to the undoped insulator which exhibits an isotropic dispersion about the $(\pi / 2, \pi / 2)$ points [23]. This implies that the electronic dispersion in the superconductor in this region of the zone may not be as closely related to the insulator as has been recently suggested [24].

We now return to Eq. (1). It is known from previous ARPES measurements that the band dispersion along $(0,0)-(\pi, \pi)$ is rather strong and doping independent [25] with an estimated $v_{F}=2.5 \times 10^{7} \mathrm{~cm} / \mathrm{sec}$ [19]. It is also known that $k_{F}$ along this direction is $0.737 \AA^{-1}$ and relatively doping independent [16]. Using these inputs, together with the strongly doping dependent $v_{\Delta}$, we can estimate the slope $\left|d \lambda^{-2} / d T\right|$ in the case of noninteracting quasiparticles $(\beta=1)$. As shown in Fig. 3b (filled circles) we find the resulting slope is of the order $6 \times 10^{-9} \AA^{-2} \mathrm{~K}^{-1}$ and is reduced by approximately $30 \%$ in going from UD75K to OD87K.

Figure $3 \mathrm{~b}$ also shows the values of $\left|d \lambda^{-2} / d T\right|$ obtained from London penetration depth measurements [6,12-15]. Although, there is considerable variation in the measured 
values of $\lambda(0)$ and low temperature $d \lambda / d T$ from one group to another, probably due to the use of different techniques, we find evidence for the following trend: the slope $d \rho_{s} / d T$ decreases with underdoping. For $\mathrm{YBa}_{2} \mathrm{Cu}_{3} \mathrm{O}_{7-\delta}$ (YBCO) this effect is weak in the British Columbia data [12], but much stronger in the Cambridge data [15]. The limited data available for Bi2212 are consistent with this trend [14]. The striking feature is that, in all cases, this trend in $d \rho_{s} / d T$ is exactly the opposite of that deduced from a theory with noninteracting quasiparticles $(\beta=1)$ using ARPES input. That is, from Fig. $3 b$, it is clear that the renormalization factor $\beta$ is considerably smaller than unity and doping dependent, a conclusion different from that inferred earlier $[4,5,7]$. To get an estimate of the doping dependence of $\beta$, we use the Bi2212 values of Ref. [14] for OD85K and UD80K samples in comparison to our own values on OD87K and UD80K, obtaining a $\beta^{2}$ of 0.32 and 0.17 , respectively. This is roughly consistent with a $\beta$ which varies as $x$, the number of doped holes, which would be the expected result from the $x$ scaling of $\rho_{s}(0)$ $[4,5]$. On the other hand, as noted earlier [5], a weaker doping dependence of $\beta$ seems to be implied by the British Columbia data. Given the difficulties of measuring the superconducting gap in YBCO by ARPES, this points to the need for further penetration depth experiments on Bi2212 samples so that a more detailed comparison to ARPES data can be made.

In conclusion, we find that the gap anisotropy of Bi2212 changes strongly as a function of doping, implying an increase in the range of the pairing interaction with underdoping. Moreover, a comparison of our data to penetration depth measurements indicates that the slope of the superfluid density is renormalized by a doping dependent factor, implying that a noninteracting picture of quasiparticle excitations around the nodes of the $d$-wave order parameter is inappropriate. This has obvious implications for other low temperature measurements in the high temperature cuprate superconductors, such as specific heat, NMR, and microwave and thermal conductivity, which are usually quantified by theories which do not take into account these renormalizations.

This work was supported by the U.S. Department of Energy, Basic Energy Sciences, under Contract No. W-
31-109-ENG-38, the National Science Foundation DMR 9624048, and DMR 91-20000 through the Science and Technology Center for Superconductivity, and the CREST of JST. J. M. is supported by the Swiss National Science Foundation, and M. R. by the Indian DST.

[1] P. W. Anderson, The Theory of Superconductivity in the High $T_{c}$ Cuprates (Princeton Univ. Pr., Princeton, 1997).

[2] C. G. Olson et al., Phys. Rev. B 42, 381 (1990).

[3] Y. J. Uemura et al., Phys. Rev. Lett. 62, 2317 (1989).

[4] P.A. Lee and X.-G. Wen, Phys. Rev. Lett. 78, 4111 (1997); 80, 2193 (1998).

[5] A. J. Millis, S.M. Girvin, L. B. Ioffe, and A. I. Larkin, J. Phys. Chem. Solids 59, 1742 (1998).

[6] W. N. Hardy et al., Phys. Rev. Lett. 70, 3999 (1993).

[7] C. Panagopoulos and T. Xiang, Phys. Rev. Lett. 81, 2336 (1998).

[8] P. Nozieres, Theory of Interacting Fermi Systems (Addison-Wesley, Reading, 1964), p. 10; A. J. Leggett, Phys. Rev. 140, A1869 (1965).

[9] J. M. Harris et al., Phys. Rev. B 54, R15 665 (1996).

[10] H. Ding et al., J. Phys. Chem. Solids 59, 1888 (1998).

[11] Ch. Renner et al., Phys. Rev. Lett. 80, 149 (1998); N. Miyakawa et al., Phys. Rev. Lett. 80, 157 (1998).

[12] D. A. Bonn et al., Czech. J. Phys. 46, 3195 (1996).

[13] S.-F. Lee et al., Phys. Rev. Lett. 77, 735 (1996). See also T. Jacobs et al., Phys. Rev. Lett. 75, 4516 (1995).

[14] O. Waldmann et al., Phys. Rev. B 53, 11825 (1996). This measurement is restricted to $T>17 \mathrm{~K}$.

[15] C. Panagopoulos, J. R. Cooper, and T. Xiang, Phys. Rev. B 57, 13422 (1998).

[16] H. Ding et al., Phys. Rev. Lett. 78, 2628 (1997).

[17] Z.-X. Shen et al., Phys. Rev. Lett. 70, 1553 (1993).

[18] H. Ding et al., Phys. Rev. B 54, R9678 (1996).

[19] H. Ding et al., Phys. Rev. Lett. 74, 2784 (1995).

[20] M. R. Norman et al., Nature (London) 392, 157 (1998).

[21] R. Fehrenbacher and M. R. Norman, Phys. Rev. B 50, 3495 (1994).

[22] S. Chakravarty, A. Sudbo, P. W. Anderson, and S. Strong, Science 261, 337 (1993).

[23] B. O. Wells et al., Phys. Rev. Lett. 74, 964 (1995).

[24] F. Ronning et al., Science 282, 2067 (1998).

[25] D. S. Marshall et al., Phys. Rev. Lett. 76, 4841 (1996). 